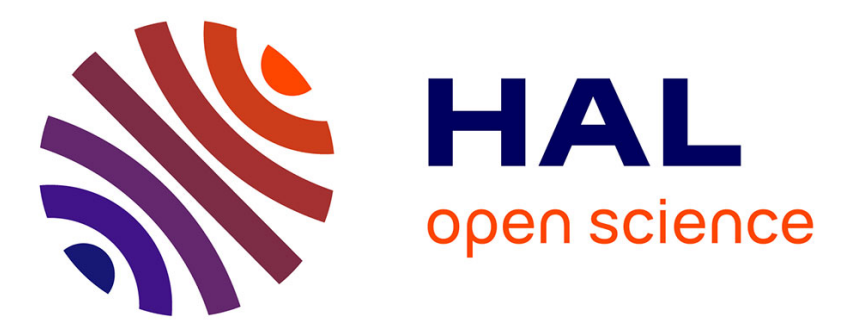

\title{
Experiment of diffraction in nonlinear optics : second harmonic generation by a nonlinear grating
}

R. Reinisch, G. Chartier, M. Neviere, M.C. Hutley, G. Clauss, J.P. Galaup, J.F. Eloy

\section{- To cite this version:}

R. Reinisch, G. Chartier, M. Neviere, M.C. Hutley, G. Clauss, et al.. Experiment of diffraction in nonlinear optics: second harmonic generation by a nonlinear grating. Journal de Physique Lettres, 1983, 44 (24), pp.1007-1012. 10.1051/jphyslet:0198300440240100700 . jpa-00232289

HAL Id: jpa-00232289

https://hal.science/jpa-00232289

Submitted on 1 Jan 1983

HAL is a multi-disciplinary open access archive for the deposit and dissemination of scientific research documents, whether they are published or not. The documents may come from teaching and research institutions in France or abroad, or from public or private research centers.
L'archive ouverte pluridisciplinaire HAL, est destinée au dépôt et à la diffusion de documents scientifiques de niveau recherche, publiés ou non, émanant des établissements d'enseignement et de recherche français ou étrangers, des laboratoires publics ou privés. 
Classification

Physics Abstracts

$42.65 \mathrm{C}-42.80 \mathrm{~F}-78.45$

\title{
Experiment of diffraction in nonlinear optics : second harmonic generation by a nonlinear grating
}

\author{
R. Reinisch $\left({ }^{*}\right)$, G. Chartier $(*)$, M. Nevière $\left({ }^{* *}\right)$, M. C. Hutley $\left({ }^{* * *}\right)$, G. Clauss $\left({ }^{*}\right)$, \\ J. P. Galaup $\left(^{+}\right)$and J. F. Eloy $\left({ }^{++}\right)$ \\ (*) Laboratoire de Génie Physique, ERA 836, CNRS, E.N.S.I.E.G., BP 46, 38402 Saint Martin \\ d'Hères, France \\ (**) Laboratoire d'Optique Electromagnétique, ERA 597, CNRS, Faculté des Sciences et Tech- \\ niques, Centre de St Jérôme, 13397 Marseille Cedex 13, France \\ (***) National Physical Laboratory, Teddington, Middlesex, U.K. \\ $\left({ }^{+}\right)$Laboratoire de Spectrométrie Physique, USMG, Domaine Universitaire, BP 68, 38402 Saint \\ Martin d'Hères, France \\ $\left({ }^{++}\right)$Centre d'Etudes Nucléaires de Grenoble, 85X, 38040 Grenoble Cedex, France
}

(Reçu le 4 juillet 1983, accepté le 2 novembre 1983)

\begin{abstract}
Résumé. - Nous décrivons une expérience où le second harmonique d'un rayonnement lumineux est obtenu à l'aide d'un réseau non linéaire, c'est-à-dire d'un réseau de diffraction réalisé sur un milieu non linéaire (qui dans notre cas est en argent). La profondeur des sillons est variable. Nous observons l'existence d'une profondeur optimale pour laquelle l'intensité du second harmonique est optimum.
\end{abstract}

\begin{abstract}
We report an experiment in which second harmonic generation is performed using a nonlinear grating, i.e. a grating impressed on a nonlinear medium (which, in our case, is silver), with variable groove depth. We observed for the groove depth the existence of an optimum value for which the second harmonic peak intensity is the greatest.
\end{abstract}

Let us consider an interface separating two media (1) and (2), see figure 1 , on which is sent a parallel incident light beam $I$ having a frequency $\omega$ and propagating in medium (1), we are interested by the light $R$ which is sent back in medium (1) by an interface which can be either a smooth surface (Fig. 1a) or a grating (Fig. 1b). If both media (1) and (2) are linear, in the optical sense of this word, the light $R$ has the same frequency as the incident light beam. If either one or the two media have non linear properties, then $R$ will have other frequency components than $\omega$. The first experiments of this kind have been performed by Bloembergen et al. [1] who have demonstrated the existence of harmonic light (frequency $2 \omega$ ) in the light sent back in medium (1). Their interface was simply a silver mirror and under that condition $R$ was a parallel beam emitted in a direction according to the Snell-Descartes laws.

Other experiments have shown that the efficiency of nonlinear optical processes, such as Surface Enhanced Raman Scattering [2] (SERS) or Second Harmonic Generation [3] (SHG), could be enhanced by the presence of rough surfaces. Among the various mechanisms which contribute to this enhancement, the Surface Plasmon Resonance (SPR) is expected to play a significant rôle [4]. 


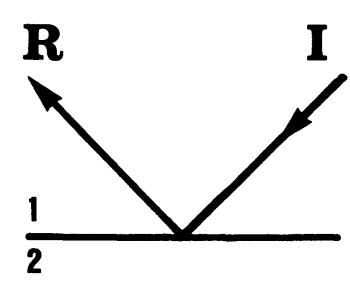

a)

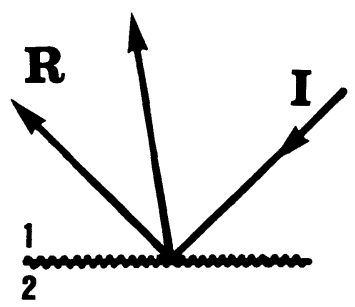

b)

Fig. 1. - When an incident light beam $I$ is sent on some interface $((a):$ smooth; $(b):$ grating), various mechanisms (scattering, diffraction, reflection) send some light $R$ back into the first medium. $R$ has usually the same frequency as $I$, except if some nonlinear properties are involved. We are interested in what happens when the interface has the shape of a diffraction grating, covered with a material having nonlinear properties (silver in our case).

Since metals are centrosymmetric, the nonlinear susceptibility of order two is zero. As a consequence, the second-order non-linearity in metals is due to metal atoms located in the very vicinity of the metal surface where there is a breaking of the inversion symmetry. This effect is not related with the shape (smooth or rough) of the entrance face of the metal.

A recent theory [5] has shown that the study of enhanced nonlinear optical effects on a surface is nothing but a special case of a new area of optics, namely "diffraction in nonlinear optics ".

The formalism developed in reference 5, since it does not consider the groove depth of the nonlinear grating (i.e. a grating impressed on a nonlinear material) as a perturbative parameter, allows the prediction of an important result : the existence, when SPR occurs; of an optimum value for the groove depth. The nonlinear conversion efficiency then takes its greatest value. The authors of reference 5 have also derived the nonlinear grating equation which determines the propagation direction of the radiated nonlinear diffracted orders.

The purpose of this Communication is to report on early results concerning an experiment of diffraction in nonlinear optics and demonstrating SHG by a so-called nonlinear grating.

The diffraction grating we used is a holographic sinusoidal grating, made on a resin; its periodicity $d$ is $5556 \AA$, its total length is $20 \mathrm{~cm}$. During fabrication the time of exposure has not been kept constant for all the points of the grating, so that the groove depth $\delta$ smoothly varies from $160 \AA$ at one edge to $700 \AA$ at the other edge of the grating. The grating has been covered first with a smooth layer of silver (thickness $\simeq 2000 \AA$ ) and then with a protecting coating of silicon oxide $\mathrm{SiO}(300 \AA)$.

The experimental set up is given in figure 2, we use a Nd-YAG laser which is Q-Switched by a saturable absorber and works at a $\lambda_{1}=1.06 \mu \mathrm{m}$ wavelength on its $\mathrm{TEM}_{\mathrm{oo}}$ mode. A $10 \mathrm{~mJ}$ light pulse is emitted each second, it has the shape of a triangle with half width of $2 \mathrm{~ns}$. The $1.06 \mu \mathrm{m}$ laser beam has a cross section of about $20 \mathrm{~mm}^{2}$, it is not focused on the grating.

The second harmonic beam is detected by a Photomultiplier (EMI $9558 \mathrm{~B}$ ). In front of the Photomultiplier was put first an IR filter (which was a dielectric mirror designed to have a $100 \%$ reflection coefficient at $1.06 \mu \mathrm{m})$. Then we placed either an interference filter centred at the harmonic wavelength $\left(\lambda_{2}=0.53 \mu \mathrm{m}\right)$ or a monochromator. In order to vary the groove depth for which the nonlinear interaction occurs, the grating could be translated parallel to itself. The polarization of the laser light incident on the grating could be fixed (TM or TE) and the angles of incidence $\theta$ and of nonlinear diffraction $\psi$ could be varied.

Before going into the detail of the data, let us first quote some useful theoretical results [5]. With these values of $\lambda_{1}$ and $d$ the only propagating diffracted order at the pump frequency $\omega$ is the specularly reflected one; the +1 diffracted order at $1.06 \mu \mathrm{m}$ is evanescent above the grating 


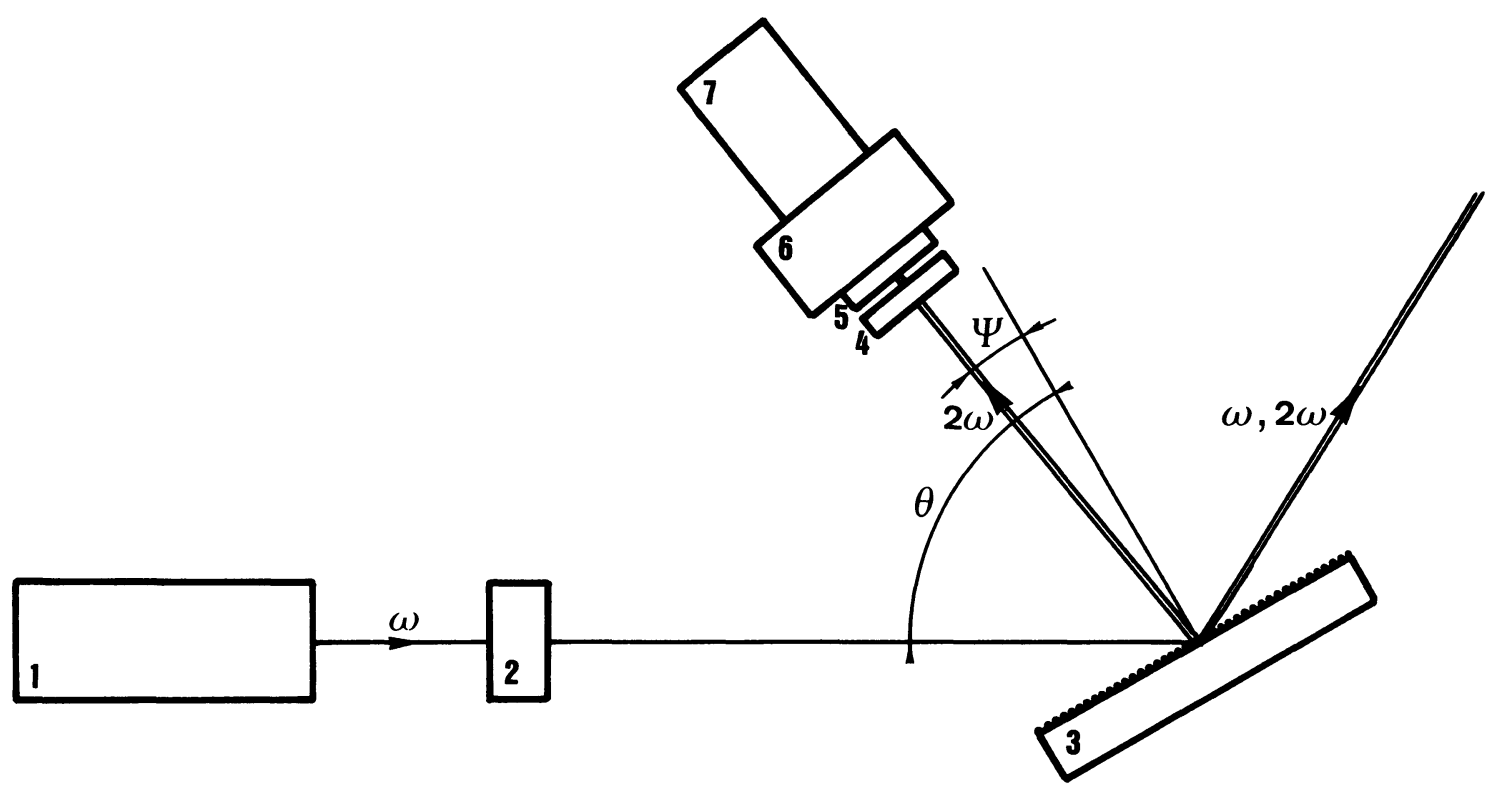

Fig. 2. - Experimental set up, 1 : Nd-YAG laser, 2 : Polarizer, 3 : Grating with variable groove depth, $4:$ IR filter, $5: \lambda_{2}$ filter, $6:$ Monochromator, $7:$ Photomultiplier.

for any value of the angle of incidence. Thus, this +1 diffracted order at $1.06 \mu \mathrm{m}$ can be resonantly excited; which means that it exists a specific value $\theta_{\text {res }}$ of $\theta$ for which SPR occurs. The calculated value of $\theta_{\text {res }}$ is $62.42^{\circ}$.

Using the rigorous electromagnetic theory of diffraction [6], it can be shown that, at the pump wavelength $\lambda_{1}$, the optimum value of $\delta$ is $\delta_{\text {opt }}\left(\lambda_{1}\right)=114 \AA$.

In the case of SHG, the nonlinear grating equation reduces to $[5,7]$

$$
\frac{d}{\lambda_{2}}\left(\sin \psi_{\mathrm{n}}-\sin \theta\right)=n
$$

where $\psi_{\mathrm{n}}$ is the nonlinear diffraction angle of the diffracted order $n$ at the second harmonic wavelength $\lambda_{2}\left(\lambda_{2}=0.53 \mu \mathrm{m}\right) n=0, \pm 1, \pm 2, \ldots$

Equation 1 shows that, at the second harmonic wavelength $\lambda_{2}=\lambda_{1} / 2$ there are only two propagating diffracted orders : the 0 and -1 orders. Since the outside medium is not dispersive, 0 orders at $\lambda_{1}$ and $\lambda_{2}$ propagate in the same direction. For the -1 order we find $\psi_{-1}=-3.87^{\circ}$.

The experiment was performed in the following way : for each groove depth $\delta$ :

a) the value $\theta_{\text {res }}$ is determined by noting the angle $\theta$ for which the intensity of the specularly diffracted order at $\lambda_{1}=1.06 \mu \mathrm{m}$ is minimum;

b) for $\theta=\theta_{\text {res }}$, the nonlinear diffraction angle $\psi$ is then varied to obtain the highest intensity, $I\left(\lambda_{2}\right)$ of the -1 diffracted order at $\lambda_{2}$.

The experimental results are the following :

- No Second Harmonic Signal was detected when the pump beam was TE polarized (magnetic field in the plane of incidence).

- The spatial distribution of the -1 diffracted order at $\lambda_{2}=0.53 \mu \mathrm{m}$ is depicted in figure 3 , whereas figure 4 is a plot of the spectral distribution of this beam (as obtained with the mono- 


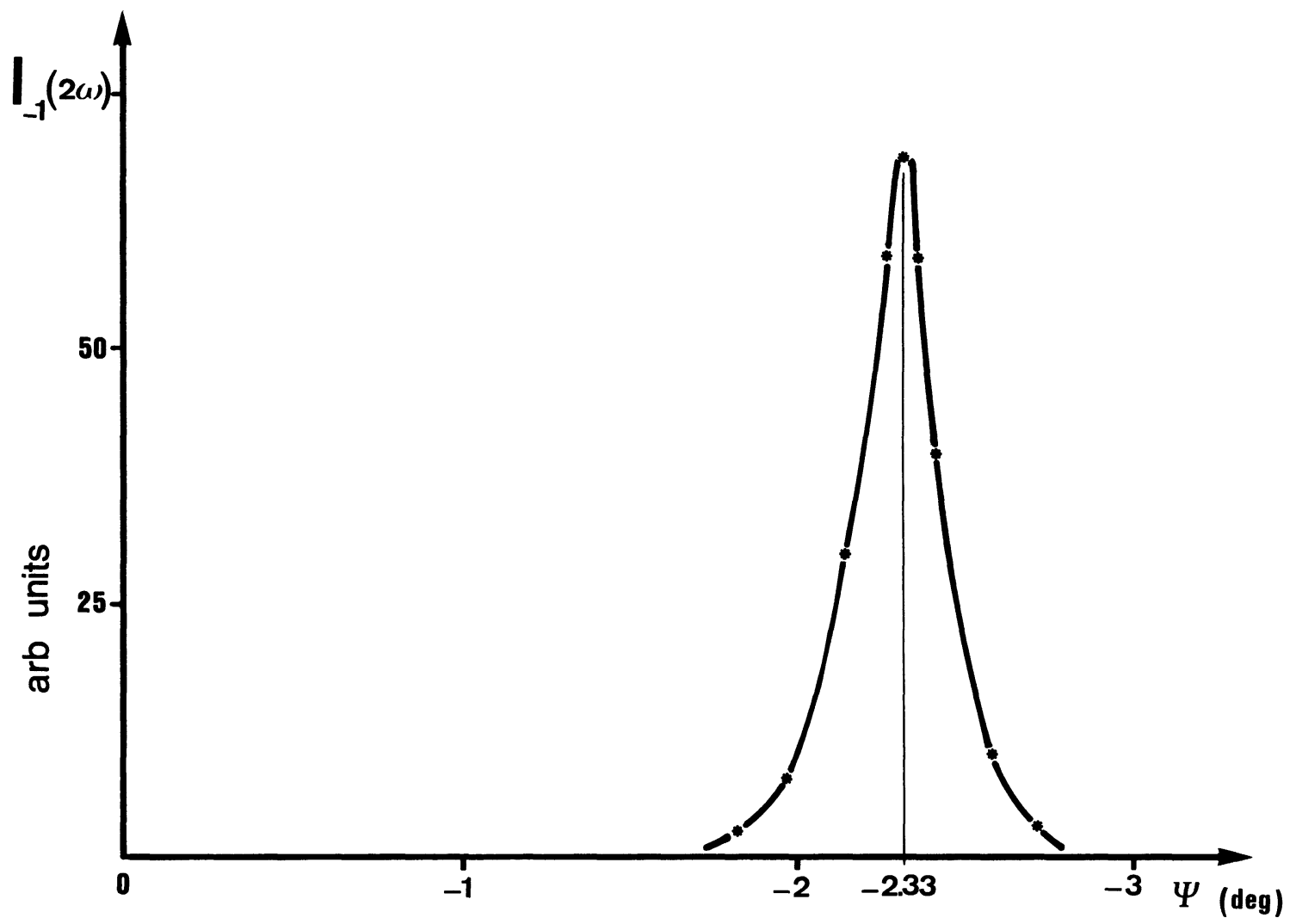

Fig. 3. - Spatial distribution of the -1 diffracted order at $\lambda_{2}=0.53 \mu \mathrm{m}$.

chromator). This figure was obtained for the value $\psi_{\mathrm{M}}$ of $\psi$ corresponding to the direction of propagation of the -1 diffracted order at the harmonic wavelength $\lambda_{2}$.

- In figure 5, we report the variation of the second harmonic peak intensity corresponding to $\psi=\psi_{\mathrm{M}}$ and $\theta=\theta_{\text {res }}$ as a function of the groove depth $\delta$ :

Figures 2 and 3 show that :

a) the detected beam in the direction $\psi_{\mathrm{M}}$ corresponds to SHG;

b) this beam is emitted in a well defined direction $\psi_{M}=-2.33^{\circ}$ which is in reasonable agreement with the calculated value $\psi_{-1}=-3.87^{\circ}$.

Figures 3 and 4 were obtained for $\delta=\delta_{\text {opt }}\left(\lambda_{2}\right)=560 \AA$. Other groove depths lead to results similar to those quoted in these two figures.

The measured value of $\theta_{\text {res }}$ is found to depend slightly on $\delta$. When $\delta$ varies from $160 \AA$ to $700 \AA, \theta_{\text {res }}$ varies from $60.67^{\circ}$ to $62.17^{\circ}$. This is in agreement with the corresponding theoretical results [5].

Finally, figure 5 clearly demonstrates the existence of an optimum value of the groove depth $\delta$ : $\delta_{\text {opt }}\left(\lambda_{2}\right)=560 \AA$ for which the second harmonic peak intensity is the greatest. It can be noticed that $\delta_{\text {opt }}\left(\lambda_{2}\right)$ is different from $\delta_{\text {opt }}\left(\lambda_{1}\right)$.

The results concerning the direction of emission and pump polarization dependence of the Second Harmonic light are different from those reported by Chen et al. [3] who find an emission which is nearly isotropic and which does not depend on the input polarization. This can be understood by noting that Chen et al. used a random rough surface which can be thought as a 


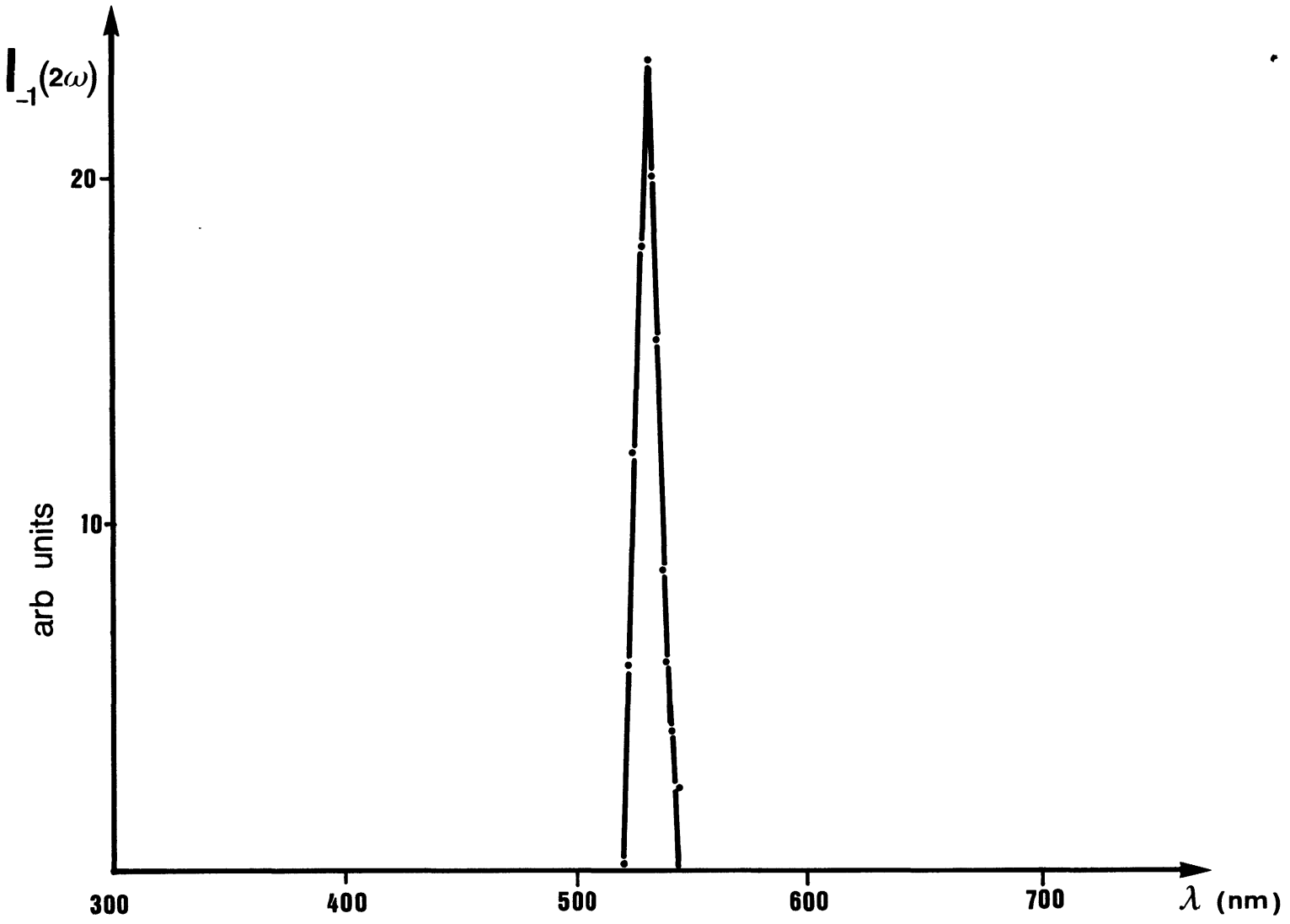

Fig. 4. - Spectral distribution of the -1 diffracted order.

superposition of an infinite number of gratings having wavevectors with different directions (and also different magnitudes). Since for each of these elementary gratings SHG is directional and depends on the pump polarization, one easily understands that the net effect of a randomly rough surface is an isotropic angular distribution and polarization free SHG [8].

In conclusion, the experimental results reported in this Communication confirm the predictions of the theory of diffraction in nonlinear optics and especially the existence of an optimum value of the groove depth of the nonlinear grating for which Second Harmonic Conversion is maximum.

\section{References}

[1] Bloembergen, N., Nonlinear Optics (Benjamin, N.Y.) 1965.

[2] Burstein, E., Chen, C. Y. and Lundquist, S., Light Scattering in Solids, Proceeding on the 2nd joint U.S.A.-U.S.S.R. Symposium, ed. J. L. Birman, H. Z. Cummins, K. K. Rebane (Plenum Press) 1979.

Schultz, S. G., Jani-Czachor, M. and van Duyne, R. P., Surface Science 104 (1981) 419.

Otтo, A., Appl. Surf. Sci. 6 (1980) 309.

FurTaK, T. E. and ReYes, J., Surf. Sci. 93 (1980) 351. 


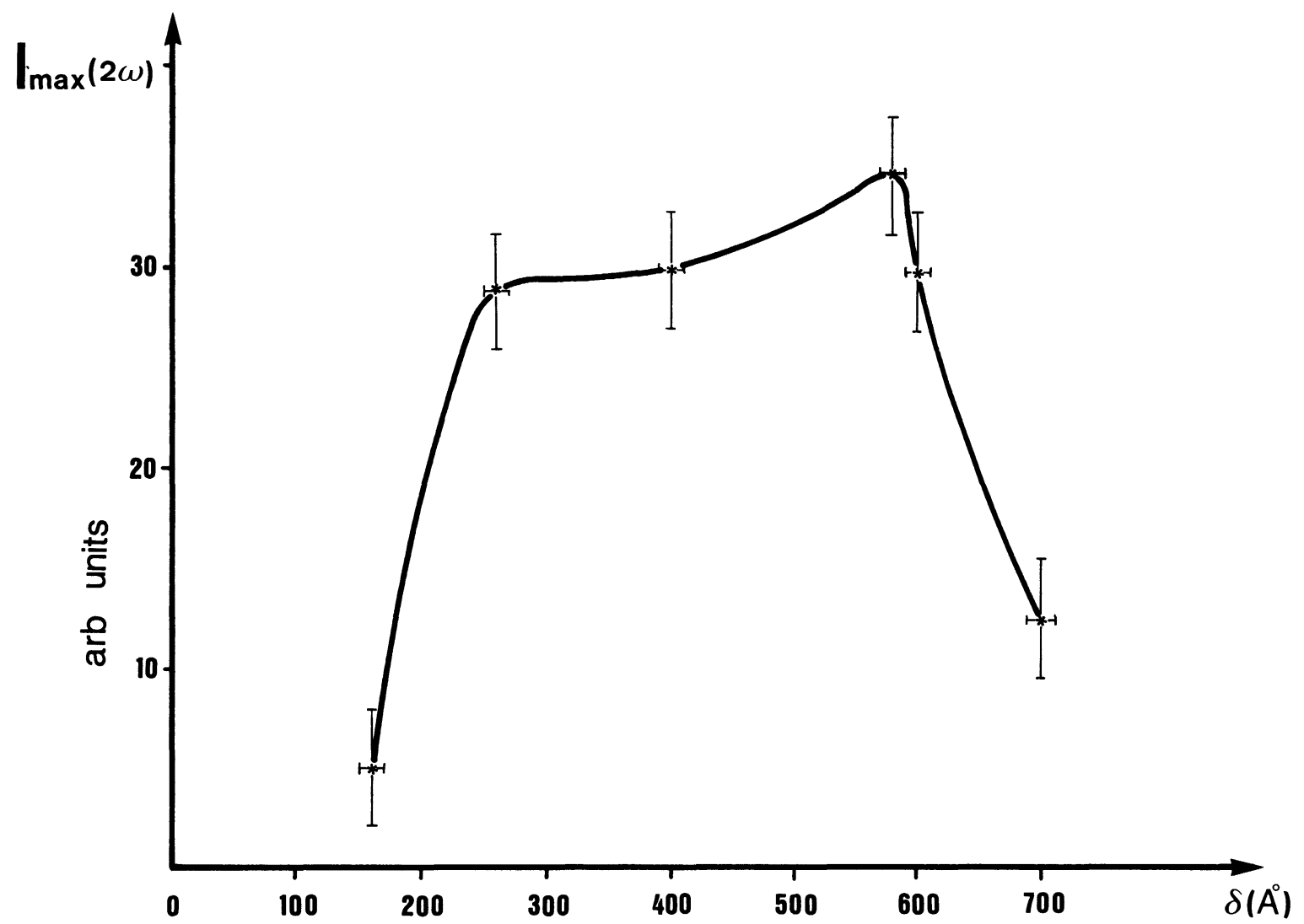

Fig. 5. - Peak intensity of the -1 diffracted order at the second harmonic frequency as a function of the groove depth $\delta$.

[3] Chen, C. K., De Castro, A. R. B. and Shen, Y. R., Phys. Rev. Lett. 46 (1981) 145.

Wokaun, A., Bergman, J. G., Heritage, J. P., Glass, A. M., Liao, P. F. and Holson, D. H., Phys. Rev. B 24 (1981) 849.

[4] McCall, S. L., Platzman, P. M. and Wolff, P. A., Phys. Lett. 77A (1980) 381.

Tsang, J. C., Kirtley, J. R. and Bradley, J. A., Phys. Rev. Lett. 43 (1979) 772.

Moskovits, M., J. Chem. Phys. 69 (1978) 4159.

EesLey, G. L., Phys. Rev. B 24 (1981) 5477.

Jha, S. S., Kirtley, J. R. and Tsang, J. C., Phys. Rev. B 22 (1980) 3973.

[5] Reinisch, R. and Neviere, M., Phys. Rev. B 28 (1983) 1870.

[6] The Electromagnetic theory of gratings, edited by PETIT, R. (Springer, Berlin) 1980 and references cited therein.

[7] To our knowledge, in the case of SHG, the nonlinear grating equation (Eq. 1) was first derived by WOKAUN et al. (see Ref. 3 of this paper).

[8] Neviere, M. and Reinisch, R., Phys. Rev. B 26 (1982) 5403. In that paper, a similar explanation has been given concerning the fact that SERS does not depend on the polarization of light. See also reference 11 of that paper. 